\title{
A COMPARATIVE STUDY BETWEEN CAROTID PLAQUE CHARACTERISTICS IN SYMPTOMATIC (TIA AND STROKE) AND ASYMPTOMATIC PATIENTS
}

\author{
Deepak Varshney1, Manish Bhagat², Rupali Gupta³, Naman Kumar Gaur, Shoaib Hussain ${ }^{5}$, Santeshwari6, Manu Gupta7, Vipul Goyal ${ }^{8}$ \\ ${ }^{1}$ Assistant Professor, Department of Radiodiagnosis, SAIMS, Indore. \\ ${ }^{2}$ Assistant Professor, Department of Radiodiagnosis, SAIMS, Indore. \\ ${ }^{3}$ DNB Postgraduate Resident, Department of Radiodiagnosis, Saifee Hospital, Mumbai. \\ ${ }^{4}$ Postgraduate Resident, Department of Radiodiagnosis, SAIMS, Indore. \\ ${ }^{5}$ Postgraduate Resident, Department of Radiodiagnosis, SAIMS, Indore. \\ ${ }^{6}$ Postgraduate Resident, Department of Radiodiagnosis, SAIMS, Indore. \\ ${ }^{7}$ Postgraduate Resident, Department of Radiodiagnosis, SAIMS, Indore. \\ ${ }^{8}$ Postgraduate Resident, Department of Radiodiagnosis, SAIMS, Indore.
}

\section{ABSTRACT}

\section{BACKGROUND}

Few cohort studies have examined the association of carotid plaque characteristics in symptomatic and apparently healthy persons. The aim of our comparative study is to evaluate the relationship of carotid plaques, surface and morphology in symptomatic and asymptomatic patients in a clinical setting.

\section{MATERIALS AND METHODS}

Carotid plaques were evaluated bilaterally with ultrasonography in 75 patients, with and without a previous stroke or coronary heart disease. In this comparative study, the subsequent incidence of stroke was investigated with respect to plaque morphology.

\section{RESULTS}

All the cases of TIA and Stroke had occurred within 2 weeks before examination. Cases of TIA and Stroke combined, represented the symptomatic group. Out of the 38 cases of TIA, 11 (29\%) cases had previous history of TIA and one patient had history of amaurosis fugax. Among 37 cases of stroke, 8 (21.6\%) cases had previous history of TIA. The age range of the cases was from 18 to 68 years with the mean age being 57.1 years. Mean age in symptomatic (TIA \& Stroke) and asymptomatic group was 56.5 years and 56.8 years respectively. $50 \%$ cases were in the age group of 60 to 69 years. Among the symptomatic cases $50.6 \%$ and among the asymptomatic cases $48.6 \%$ were in the age range of 60 to 69 years. Among the 58 plaques seen in cases of TIA and Stroke ipsilateral to hemisphere involved, $44(75.86 \%)$ were heterogeneous, whereas among the 9 plaques seen in asymptomatic cases only $1(11.11 \%)$ was heterogeneous. The association of heterogeneous plaque with the presence of symptoms was highly significant. $(\mathrm{Z}=5.44, \mathrm{p}<0.01)$. There was more difference in the prevalence of homogenous (Fig. 2) and heterogenous plaque between cases of TIA and Stroke. The prevalence of irregular surface of plaques (32) (Fig. 3) was significantly ( $p$ value $<0.01$ ) more in symptomatic cases (55.17\%) as compared to asymptomatic cases $(11.11 \%)$. Whereas the prevalence of irregular surface of plaque was almost the same in cases of TIA and stroke. No plaque of the S3 type (pocketed) was found in this study.

\section{CONCLUSION}

Prevalence of heterogeneous and irregular surface of plaque ipsilateral to the brain hemisphere involved was statistically more significant in symptomatic cases. A direct relationship was seen between the prevalence of heterogeneous plaque and irregular surface of plaque with the severity of stenosis.

\section{KEYWORDS}

Carotid Plaque Characteristics, Carotid Ultrasonography and Doppler Study.

HOW TO CITE THIS ARTICLE: Varshney D, Bhagat M, Gupta R, et al. A comparative study between carotid plaque characteristics in symptomatic (TIA and Stroke) and asymptomatic patients. J. Evolution Med. Dent. Sci. 2017;6(48):3717-3721, D0I: 10.14260/Jemds/2017/802

\section{BACKGROUND}

Cerebrovascular diseases are among the most frequent cause of mortality and morbidity in the elderly and are a frequent concomitant pathology in patients with cardiovascular

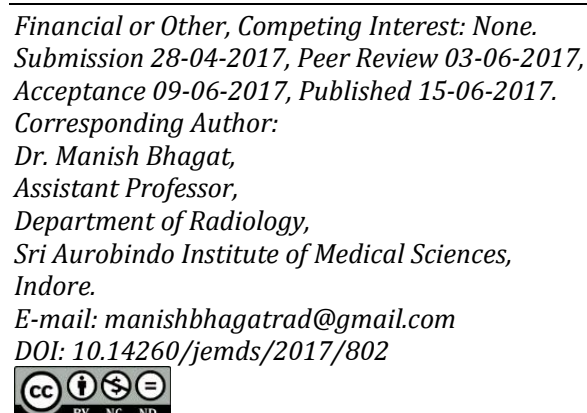

problems (i.e. coronary artery disease). Cerebrovascular disease (stroke) is the third leading cause of death in the United States, accounting for approximately 400,000 new cases diagnosed each year and over 163,000 deaths in 2002.[1] Men tend to have more frequent strokes at a younger age $(<75$ years) but overall, women have more strokes than men. At least $15-20 \%$ of all ischaemic strokes are attributed to carotid artery atherosclerosis.[2] Furthermore, it is estimated that $5-10 \%$ of individuals over 65 years of age have asymptomatic carotid artery atherosclerosis with at least $50 \%$ artery stenosis. ${ }^{[3,4]}$ Single or multiple segment atherosclerotic lesion of the extracranial cerebral arteries in ischaemic stroke syndromes seen in over half of all cases. Stenosis of the carotid arteries are responsible for many TIAs and may precede a complete stroke in half of these patients. 
The risk of stroke associated with symptomatic carotid artery disease is related to the severity of the carotid artery stenosis.

Nowadays duplex ultrasonography has become the investigation of first choice because it provides detailed information on the localisation and degree of stenosis, flow dynamics and condition of vessel wall. It is, however, a highly operator-dependent investigation technique and may produce suboptimal quality, e.g. in patients with short neck, high carotid bifurcation, or highly calcified plaques.

The extent, location, and characteristics of atherosclerotic plaque in the common carotid artery (CCA) and internal carotid artery (ICA) should be documented with grey-scale imaging. The vessels should be imaged as completely as possible, with caudal angulation of the transducer in the supraclavicular region and cephalic angulation at the level of the mandible. Colour Doppler imaging should be performed to detect areas of abnormal blood flow that require Doppler spectral analysis. Pulsed-wave (PW) Doppler spectral analysis should be performed, and the velocity of blood flow in the mid-CCA and proximal ICA as well as proximal to, at, and immediately distal to the diseased areas should be measured.[5]

The aim of our prospective study is to evaluate the complementary role of colour-coded duplex U/S in the functional and morphological assessment of small atherosclerotic plaques and to define the extent, location and characterisation of the plaque in a clinical setting.

Heterogeneous and irregular surface plaques are known to be associated with a higher risk of stroke.[6] The detection of plaque ulceration using $\mathrm{U} / \mathrm{S}$ is controversial.[7]

With the above background, this study was planned to systematically document CDU findings in cases of stroke and TIA and to evaluate the complimentary role of colour-coded duplex U/S i.e. the functional and morphological assessment obtained by duplex U/S.

\section{MATERIALS AND METHODS}

A comparative study on the role of carotid CDU in patients of TIA and ischaemic stroke was carried out in the Department of Radiodiagnosis, SAIMS, Indore from January 2015 to June 2015.

\section{Selection of Cases}

Inclusion Criteria

Unilateral classic TIA or ischaemic stroke.

\section{Exclusion Criteria}

Cases were excluded if they had stroke or TIA that was bilateral, stroke or TIA with undetermined hemispheric involvement or brainstem involvement only.

Study group comprised of all patients with a clinical diagnosis of unilateral classic TIA or ischaemic stroke (according to inclusion criteria) referred to the Radiodiagnosis Department from January 2015 to June 2015. The total sample size was 75 patients in this study and it was taken conveniently. Patients were referred from the Medicine Department of Shri Aurobindo Medical College, Indore. In all cases CDU examination was carried out within 2 weeks of sustaining stroke or TIA. Control group comprised of 35 age and sex matched adults who were asymptomatic and essentially normal on detailed physical examination, with no known risk factors for TIA and stroke.

All relevant data including compete clinical examination and relevant information was entered in a predesigned proforma (Annexure -1).

The following definitions were adhered to in selecting the study cases.

\section{Classic TIA}

Classic TIA was defined as an abnormal focal neurologic deficit lasting less than 24 hours (usually 5-20 min.).

\section{Stroke}

A completed ischaemic stroke presented with an abrupt onset of a focal neurologic deficit and typically evolving to a maximum deficit within few hours, often the patient awakens with a completed deficit. A completed stroke is sometimes heralded by one or more TIAs in the preceding days or months. The ischaemic stroke is of the thrombotic or embolic type.

\section{The Patients were Studied under the following Groups- Group I}

Patients with a diagnosis of clinical TIA.

\section{Group II}

Patients with a clinical diagnosis of completed ischaemic stroke.

\section{Group III}

Control cases i.e. asymptomatic cases.

This study is being conducted at the Department of Radiodiagnosis, SAIMS, Indore.

\section{Equipment used in the Study}

USG and duplex and colour Doppler study was performed on PHILIPS IU-22.

\section{Criteria for Assessment of Carotid Arteries on Colour Duplex Ultrasound}

1. Extent of Plaque: The length of the vessel (cephalocaudal extent in $\mathrm{mm}$ ) affected by the plaque, which was determined with images of the carotid arteries in longitudinal axis.

2. Thickness of Plaque: The maximum thickness of plaque on transverse image was recorded.

3. Location of plaque: The situation of the plaque within the carotid artery.

4. Characterisation of plaque: Homogeneous plaque (H1): Plaque was classified as homogeneous when echo pattern was uniform and the plaque had a smooth surface. Heterogeneous plaque (H2): Plaque was classified as heterogeneous when one or more focal sonolucent areas were present. The presence of calcification was ignored and was not used in classifying the plaque.

5. Plaque surface characteristics Surface characteristics of the plaque were qualitatively assessed into three categories. 
Smooth (S1)- A continuous boundary.

Irregular (S2)- An uneven pitted boundary.

Pocketed (S3)- A crater like defect with sharp margins.

\section{Methods of Statistical Analysis}

Statistical analysis was done for the following parameters.

The presence, character and surface features of plaque were compared between symptomatic and asymptomatic cases using Z-test of significance.

\section{RESULTS}

In the present comparative study, a total of 110 cases were analysed.

Table I shows the group wise distribution of these cases.

\begin{tabular}{|c|c|}
\hline TIA (Gr. - I) & $38(34.5)$ \\
\hline Stroke (Gr. - II) & $37(33)$ \\
\hline Asymptomatic (Gr. - III) & $35(31.8)$ \\
\hline Total & $\mathbf{1 1 0 ( 1 0 0 )}$ \\
\hline
\end{tabular}

Table I. Group wise Distribution of Cases ( $n=110$ cases)

Figures in parenthesis indicate percentage.

All the cases of TIA and Stroke had occurred within 2 weeks before examination. Cases of TIA and Stroke combined, represented the symptomatic group. Out of the 38 cases of TIA, $11(29 \%)$ cases had previous history of TIA and one patient had history of amaurosis fugax. Among 37 cases of stroke, 8 (21.6\%) cases had previous history of TIA.

\begin{tabular}{|c|c|}
\hline Group & No. of cases \\
\hline
\end{tabular}

\begin{tabular}{|c|c|c|c|c|c|c|c|c|c|}
\hline \multirow{2}{*}{$\begin{array}{l}\text { Age Group } \\
\text { in years }\end{array}$} & \multicolumn{2}{|c|}{$\begin{array}{c}\text { TIA } \\
(n=38)\end{array}$} & \multicolumn{2}{|c|}{$\begin{array}{l}\text { Stroke } \\
(n=37)\end{array}$} & \multirow{2}{*}{$\begin{array}{c}\begin{array}{c}\text { TIA and Stroke } \\
(n=75)\end{array} \\
\text { Total }\end{array}$} & \multicolumn{3}{|c|}{$\begin{array}{l}\text { Asymptomatic } \\
(n=35)\end{array}$} & \multirow{2}{*}{$\begin{array}{c}\begin{array}{c}\text { Symptomatic and } \\
\text { Asymptomatic }(\mathrm{n}=110)\end{array} \\
\text { Total }\end{array}$} \\
\hline & $\mathbf{M}$ & $\mathbf{F}$ & $\mathbf{M}$ & $\mathbf{F}$ & & $\mathbf{M}$ & $\mathbf{F}$ & Total & \\
\hline$<39$ & 1 & - & 1 & - & $2(2.6)$ & 1 & - & $1(2.8)$ & $3(2.7)$ \\
\hline $40-49$ & 10 & 1 & - & - & $11(14.6)$ & 4 & 1 & $5(14.2)$ & $16(14.5)$ \\
\hline $50-59$ & 10 & 1 & 12 & 1 & $24(32)$ & 11 & 1 & $12(34.2)$ & $36(32.7)$ \\
\hline $60-69$ & 12 & 3 & 19 & 4 & $38(50.6)$ & 17 & - & $17(48.6)$ & $55(50.0)$ \\
\hline Total & 33 & 5 & 32 & 5 & $75(100)$ & 33 & 2 & $35(100)$ & $110(100)$ \\
\hline
\end{tabular}

Figures in parenthesis denotes percentage.

Table II shows that the age range of the cases was from 18 to 68 years with the mean age being 57.1 years. Mean age in symptomatic (TIA \& Stroke) and asymptomatic group was 56.5 years and 56.8 years respectively. $50 \%$ cases were in the age group of 60 to 69 years. Among the symptomatic cases $50.6 \%$ and among the asymptomatic cases $48.6 \%$ were in the age range of 60 to 69 years.

\begin{tabular}{|c|c|c|c|c|}
\hline Plaque Type & TIA & Stroke & TIA and Stroke & Asymptomatic \\
\hline Homogeneous (H1) & $8(27.58)$ & $6(20.68)$ & $14(24.14)$ & $8(88.88)$ \\
\hline Heterogeneous (H2) & $21(72.4)$ & $23(79.31)$ & $44(75.86)$ & $1(11.11)$ \\
\hline Total plaque & $\mathbf{2 9 ( 1 0 0 )}$ & $\mathbf{2 9 ( 1 0 0 )}$ & $\mathbf{5 8 ( 1 0 0 )}$ & $\mathbf{9 ( 1 0 0 )}$ \\
\hline \multicolumn{5}{|r|}{ Table III } \\
\hline
\end{tabular}

$\mathrm{Z}=5.44 ; \mathrm{p}<0.01$. Figures in parenthesis indicate percentage.

Table III shows that among the 58 plaques seen in cases of TIA and Stroke, 44 (75.86\%) were heterogeneous (Fig. 1) whereas among the 9 plaques seen in asymptomatic cases only $1(11.11 \%)$ was heterogeneous. The association of heterogeneous plaque with the presence of symptoms was highly significant. $(\mathrm{Z}=5.44, \mathrm{p}<0.01)$. There was more difference in the prevalence of homogenous (Fig. 2) and heterogenous plaque between cases of TIA and Stroke.

\begin{tabular}{|c|c|c|c|}
\hline \multirow{2}{*}{ Group } & \multicolumn{2}{|c|}{ Plaque Surface } & \multirow{2}{*}{ Total } \\
\cline { 2 - 3 } & Smooth: S1 & Irregular: S2 & \\
\hline TIA & $14(48.27)$ & $15(51.72)$ & $29(100)$ \\
\hline Stroke & $12(41.37)$ & $17(58.62)$ & $29(100)$ \\
\hline TIA and Stroke & $26(44.82)$ & $32(55.17)$ & $58(100)$ \\
\hline Asymptomatic & $8(88.88)$ & $1(11.11)$ & $9(100)$ \\
\hline \multicolumn{3}{|c|}{ Table IV } \\
\hline
\end{tabular}

$\mathrm{Z}=3.575 ; \mathrm{p}<0.01$.

Figures in parenthesis indicate percentage.
Table IV shows that the prevalence of irregular surface of plaques (32)(Fig. 3) was significantly ( $p$ value $<0.01$ ) more in symptomatic cases $(55.17 \%)$ as compared to asymptomatic cases $(11.11 \%)$. Whereas the prevalence of irregular surface of plaque was almost the same in cases of TIA and stroke. No plaque of the S3 type was found in this study.

\section{DISCUSSION}

Detection and quantification of carotid artery stenosis is important as stenosis of carotid artery is a common and predisposing cause of stroke. Atherosclerotic plaque responsible for stenosis usually involves the proximal ICA. The endarterectomy trials established $60 \%$ to $70 \%$ diameter reduction as clinically significant levels of ICA stenosis, above which CEA is beneficial. Recent data from NASCET study showed modest stroke benefit from CEA at 50\% diameter ICA stenosis but only when the patient life expectancy was long \& the rate of operative morbidity and mortality was low.

Studies have shown that DU is as good as or even better than angiography in the quantification of stenotic lesions. 
Moreover, assessment of plaque morphology by DU is helpful in identifying plaque such as heterogeneous or ulcerative which can increase the risk of thromboembolism. DU is noninvasive, less costly as compared to the angiography and therefore has now emerged as a new "gold standard". Thus, DU evaluation plays a major role in carotid occlusive lesions as early and accurate identification can often benefit the patients by surgical intervention.

\section{Characteristics of Population Studied}

It was ensured in this study that the number of cases in all the three groups i.e. TIA, stroke and asymptomatic were nearly the same in number i.e. 38,37 \& 35 respectively. Among the symptomatic cases (TIA and Stroke) 19 (25.3\%) had previous history of TIA. One case of TIA had a history of amaurosis fugax, TIA is an important clue that arterial disease is present in the cerebral vasculature. In strokes ascribed to carotid disease, at least $60 \%$ were preceded by TIA, it has also been seen that TIAs are followed by stroke within 5 years in $33 \%$ of patients, with the greatest risk occurring during first two weeks after TIA. In the present study, the mean age of symptomatic cases was 56.5 years and that of asymptomatic cases was 56.8 years. Majority of the cases were 60-69 years old, symptomatic $50.0 \%$ and asymptomatic $48,6 \%$.

II. Relationship between Carotid Plaque and Symptoms

A. Character of Carotid Plaque and Symptoms

A total of 58 plaques were seen ipsilateral to the brain hemisphere involved in the symptomatic group, out of which $44 \quad(75.86 \%)$ were heterogeneous and 14 (24.14\%) were homogenous. In the asymptomatic group, 9 plaques were present out of which 1 (11.11\%) was heterogeneous. The association of heterogeneous plaque with symptomatic cases was highly significant $(\mathrm{p}<0.01)$. Our observation is comparable to that of others mentioned below who have also shown heterogenous plaques to be statistically significantly associated with symptoms of hemispheric ischaemia.

In the present study, we found no difference in the prevalence of homogenous and heterogenous plaques between cases of TIA and stroke. Reilley et al[8] in 1983 also showed hardly any difference between the prevalence of heterogeneous plaque in TIA and stroke. Contrary to this, Polak et al[9] in 1993 reported a strong association between heterogeneous plaque and stroke but this relationship was not so strong between heterogeneous plaque and TIA.

In contrast to all the above observation by various authors, Bluth et al[10] in 1986 noted that the heterogeneous group had only a slightly greater incidence of symptoms (65\%) than the homogenous group (52\%). They relate this finding to the fact that their study population consisted of patients who had flow restricting lesions as assessed by sonography and confirmed by digital vascular imaging.

\section{B. Carotid plaque surface and symptoms}

Among the symptomatic cases, irregular plaque surface was present in 32 (55.17\%) cases whereas among the asymptomatic cases the plaque surface was irregular only in $1(11.11 \%)$ case. This finding was statistically significant $(\mathrm{p}<0.01)$. There was hardly any difference between the prevalence of irregular surface plaques between cases of TIA and stroke. No S3 type plaque was detected in this study. Polak et al[9] in 1993 also found that there was a significantly increased likelihood of stroke when the lesion surface was markedly irregular and surface irregularity was also related to a significantly greater prevalence of TIA. Lannuzzi et al in 1995 showed that irregular plaque surfaces were significantly associated with recent TIA as compared with combined smooth and pocketed plaque.

The findings of this Study can be summarised as follows-

- Among the 58 plaques seen in the symptomatic cases (ipsilateral to the hemisphere involved) 44 (75.86\%) were heterogeneous whereas among the 9 plaques seen in asymptomatic cases only 1 (11.11\%) was heterogeneous. This difference was statistically significant.

- $\quad$ Prevalence of irregular surface of plaque (ipsilateral to brain hemisphere involved) was $32 / 58(55.17 \%)$ in the symptomatic cases of TIA and stroke as compared to $1 / 9$ $(11.11 \%)$ in asymptomatic cases.

- The prevalence of heterogeneous plaques increased from $37.93 \%$ in the $1-39 \%$ stenosis range to $100 \%$ in the $80-99 \%$ stenosis range whereas the prevalence of homogenous plaques decreased from $62.7 \%$ in the 1 $39 \%$ stenosis range to $5.8 \%$ in the $60-79 \%$ stenosis range. This difference was statistically significant.

- There was a statistically significant increase in number of plaques with irregular surface from $16.9 \%$ in cases with $1-39 \%$ stenosis range to $100 \%$ in cases with 80 $99 \%$ stenosis range as compared to the number of plaques with smooth surface which showed a steady decline from $84.48 \%$ in $1-39 \%$ stenosis range to $5.8 \%$ in $60-79 \%$ stenosis range.

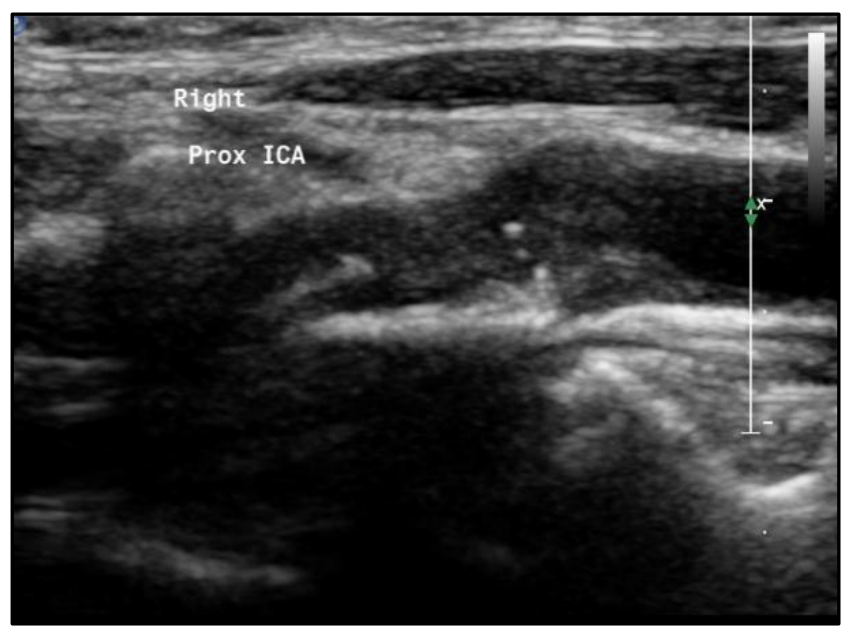

Figure 1. Longitudinal B-mode Scan shows a Heterogeneous Plaque on the Anterior Wall of the Right Prox ICA 


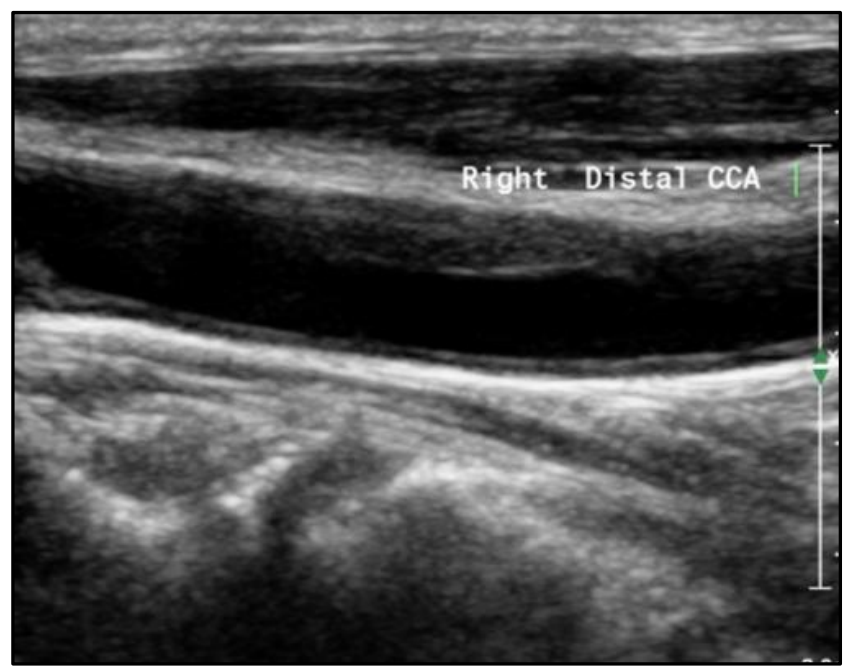

Figure 2. Longitudinal B-mode Scan shows a Homogenous Mildly Hyperechoic Plaque on the Anterior Wall of the Right Distal CCA.

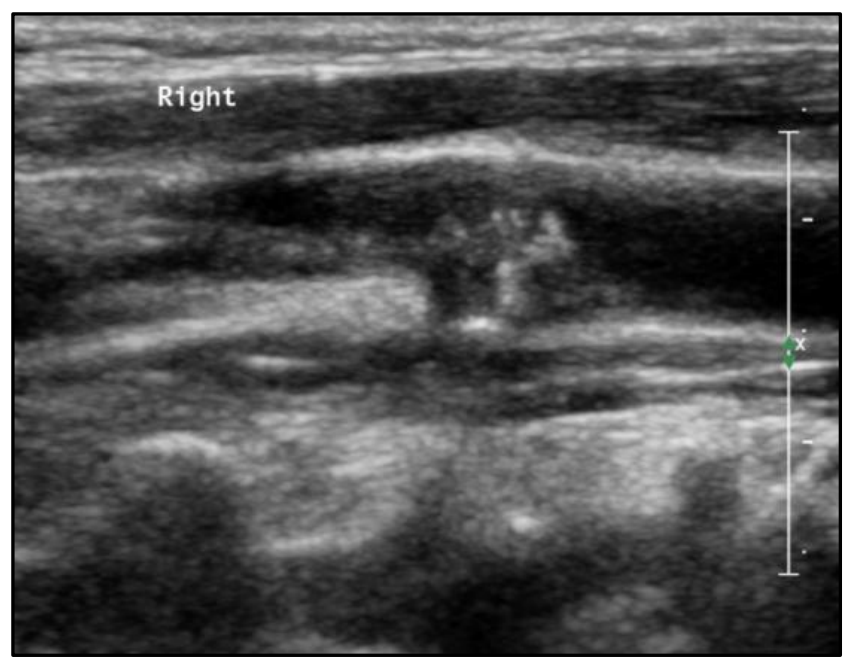

Figure 3. Longitudinal B-mode Scan shows an Ulcerated Plaque on the Posterior Wall of the Right CCA Bulb

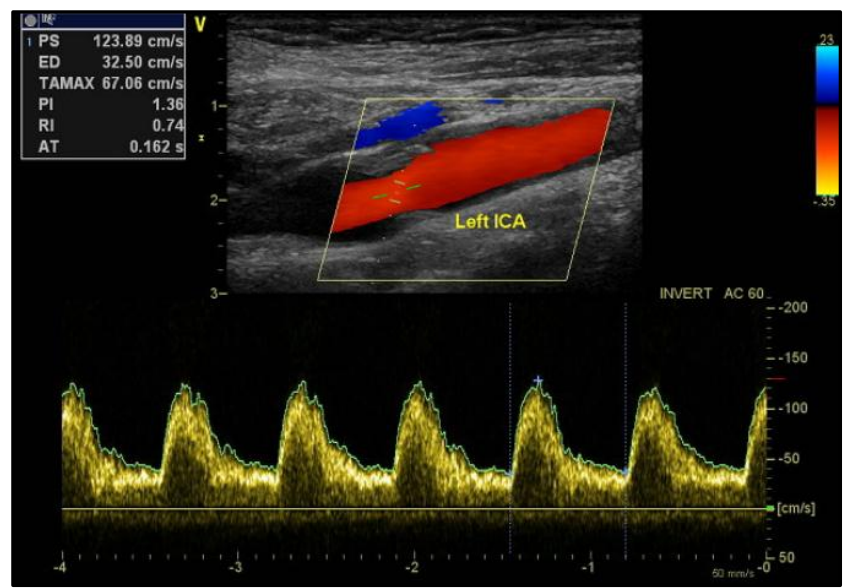

Figure 4. Longitudinal U/S scan with power Doppler imaging shows an Ulcerated Plaque at the level of the Left Carotid Bifurcation that extends to the Origin of the ICA.

\section{CONCLUSION}

We concluded that prevalence of heterogeneous and irregular surface of plaque ipsilateral to the brain hemisphere involved was statistically more significant in symptomatic cases and direct relationship was seen between the prevalence of heterogeneous plaque and irregular surface of plaque with the severity of symptoms and stenosis. CDU is not only noninvasive, widely available, accurate in quantifying stenosis but also helps in characterisation of plaque.

\section{REFERENCES}

[1] Kochanek KD, Smith BL. Deaths: preliminary data for 2002. Natl Vital Stat Rep 2004;52(13):1-47.

[2] Petty GW, Brown RD, Whisnant JP, et al. Ischemic stroke subtypes: a population-based study of incidence and risk factors. Stroke 1999;30(12):25136.

[3] Mineva PP, Manchev IC, Hadjiev DI. Prevalence and outcome of asymptomatic carotid stenosis: a population-based ultrasonographic study. Eur J Neurol 2002;9(4):383-8.

[4] Risk of stroke in the distribution of an asymptomatic carotid artery. The European carotid surgery trialists' collaborative group. Lancet 1995;345(8944):209-12.

[5] Grant EG, Barr LL, Borgstede J, et al. ACR guideline for the performance of an ultrasound examination of the extracranial cerebrovascular system. Reston, Va: American College of Radiology 2002:577-80.

[6] Steinke W, Hennerici M, Rautenberg W, et al. Symptomatic and asymptomatic high grade carotid stenosis in Doppler color-flow imaging. Neurology 1992;42(1):131-8.

[7] Carroll BA. Carotid ultrasound. Neuroimaging Clin of North Am 1996;6(4):875-97.

[8] Polak JF, O'Leary DH, Kronmal RA, et al. Sonographic evaluation of carotid $J$ artery atherosclerosis in elderly: relationship of disease severity to stroke and transient ischemic attack. Radiology 1993;188(2):36370.

[9] Lannuzzi A, Wilcosky T, Mercuri M, et al. Ultrasonographic correlates of carotid atherosclerosis in transient ischemic attack and stroke. Stroke 1995;26(4):614-9.

[10] Wilterdink JL, Feldmann E, Easton JD, et al. Optimizing the performance of carotid duplex ultrasound in evaluating carotid endarterectomy candidates: a patient outcome rather than accuracy-based approach. Stroke 1995;26:170. 